June 1993

UMDGR-93-204

\title{
ON THE CONSTRAINT ALGEBRA OF DEGENERATE RELATIVITY
}

\author{
Joseph D. Romano円 \\ Department of Physics \\ University of Maryland, College Park, MD 20742
}

\begin{abstract}
As shown by Ashtekar in the mid 80's, general relativity can be extended to incorporate degenerate metrics. This extension is not unique, however, as one can change the form of the hamiltonian constraints and obtain an alternative degenerate extension of general relativity that disagrees with Ashtekar's original theory when the triads vectors are degenerate. In this paper, the constraint algebra of a particular alternative theory is explicitly evaluated and compared with that of Ashtekar's original degenerate extension. A generic classification of the difference between the two theories is given in terms of the degeneracy and surface-forming properties of the triad vectors. (This classification is valid when the degeneracy and surface-forming properties of the triad vectors is the same everywhere in an open set about a point in space.) If the triad vectors are degenerate and surface-forming, then all the secondary constraints of the alternative degenerate extension are satisfied as a consequence of the primary constraints, and the constraints of this theory are weaker than those of Ashtekar's. If the degenerate triad vectors are not surface-forming, then the first secondary constraint of the alternative theory already implies equivalence with Ashtekar's degenerate extension. What happens when the degeneracy and surface-forming properties of the triad vectors change from point to point is an open question.
\end{abstract}

PACS: $04.20,03.50$

\footnotetext{
${ }^{1}$ Address after September 1, 1993: Physics Department, The University of Utah, 201 James Fletcher Building, Salt Lake City, Utah 84112
} 


\section{Introduction}

General relativity can be extended to incorporate degenerate metrics. Indeed, as shown by Ashtekar [1] in the mid 80's, the hamiltonian constraints of general relativity simplify when expressed in terms of a complex $S O(3)$ connection and a density-weighted spatial triad. The constraints are polynomial in these canonical variables, and the theory is welldefined even if the spatial metric is degenerate. Witten [2] used this result to quantize $2+1$ gravity, and Horowitz [3] has shown that by extending general relativity to include degenerate metrics, topology change is allowed classically. In fact, much of the renewed interest in the non-perturbative canonical quantization program for $3+1$ gravity is due to Ashtekar's degenerate extension of general relativity [四].

More recently, Jacobson and Romano [5] have shown that there exists a natural alternative choice for the form of the hamiltonian constraints that leads to a theory that agrees with general relativity (GR) for non-degenerate metrics, but differs in the degenerate sector from Ashtekar's original degenerate extension. The Poisson bracket (PB) algebra of the alternative constraints fails to close in the degenerate sector due to structure functions that involve the inverse of the spatial triad. The alternative theory has to be supplemented with an apparently infinite number of secondary constraints, which together with the primary constraints are shown to be first class. All of these constraints are implied by, but do not imply, Ashtekar's original form of the constraints, and so give rise to a different degenerate extension of GR.

One of the most puzzling and unfamiliar features of the alternative degenerate extension is its apparently infinite number of secondary constraints. It would seem that such a theory, with an infinite number of constraints per space point, would have no local degrees of freedom. Yet, as mentioned above and as shown in [5], the phase space of the alternative theory is actually larger than the phase space of Ashtekar's theory. One can write down solutions to all the secondary constraints that fail to be solutions to Ashtekar's original constraints. This suggests that the infinite number of constraints of the alternative theory are not all independent, but without an explicit expression for the higher-order secondary constraints, one does not know how to do the counting.

The purpose of this paper is to resolve this puzzle. We will find explicit expressions for all the higher-order secondary constraints. [? These expressions can be written in terms of nested commutators of the triad vectors contracted with the vector constraint of Ashtekar's original

\footnotetext{
${ }^{2}$ Since we will be interested only in obtaining a closed PB algebra for the alternative degenerate extension, we will evaluate the PBs modulo previous constraints. We will not find explicit expressions for the structure functions of this theory.
} 
degenerate extension. This new result will enable us to show that the "infinite" tower of secondary constraints for the alternative theory is not infinite, but actually collapses to a finite number of constraints. We will be able to generically classify the difference between Ashtekar's original degenerate extension of GR and the alternative degenerate extension in terms of the degeneracy and surface-forming properties of the triad vectors. In particular, we shall see that if the triad vectors are degenerate and surface-forming, then all the secondary constraints of the alternative degenerate extension are satisfied as a consequence of the primary constraints, and the constraints of this theory are weaker than those of Ashtekar's. If the degenerate triad vectors are not surface-forming, then the first secondary constraint of the alternative theory already implies equivalence with Ashtekar's degenerate extension. What happens when the degeneracy and surface-forming properties of the triad vectors change from point to point is an open question.

The rest of this paper is organized as follows: In section 2, I will briefly review the hamiltonian formulation of Ashtekar's original degenerate extension of GR [1]. My intent in this section is to give just enough detail to write down the PB algebra of the constraints. Readers interested in a more thorough discussion of Ashtekar's theory, and Ashtekar variables in general, should see [4] and references mentioned therein. In section 3, I will describe the alternative degenerate extension of GR originally discussed in [5]. I will reproduce many of results found in [5], but using a different notation (triads versus spinors) and from a slightly different point of view. For instance, the method I use to calculate the PBs for the alternative theory yields an explicit expression for all the higher-order secondary constraints - expressions that were lacking in [5]. In section 4, I will give a generic classification of the difference between the two degenerate extensions of GR and show how the apparent infinite tower of secondary constraints for the alternative theory collapses to a finite number of constraints. As mentioned above, this classification is valid when the degeneracy and surface-forming properties of the triad vectors is the same everywhere in an open set about a point in space. In section 5 , I briefly summarize the main results and conclude by raising some questions regarding those cases where the degeneracy and surface-forming properties of the triad vectors change from point to point. An appendix gives details of the calculation of the PBs for the alternative degenerate extension discussed in section 3 .

\section{Ashtekar's original degenerate extension of GR}

In Ashtekar's original degenerate extension of GR [1], the phase space variables consist

\footnotetext{
${ }^{3}$ This classification is valid when the degeneracy and surface-forming properties of the triad vectors is the same everywhere in an open set about a point in space.
} 
of a complex $S O(3)$ connection $A_{a}^{i}$ and a density-weighted $(+1)$ spatial triad $E_{i}^{a}$. Both are defined on a 3-manifold $\Sigma$. (In what follows, $a, b, c, \cdots$ denote spatial indices, while $i, j, k, \cdots$ denote internal $S O(3)$ indices.) These variables are subject to the constraints

$$
\begin{aligned}
\mathcal{G}_{i} & \equiv \mathcal{D}_{a} E_{i}^{a}=0 \\
\mathcal{S} & \equiv \epsilon^{i j}{ }_{k}^{a} E_{i}^{b} F_{a b}^{k}=0 \\
\mathcal{V}_{a} & \equiv E_{j}^{b} F_{a b}^{j}=0
\end{aligned}
$$

where $\mathcal{D}_{a}$ is the gauge-covariant derivative operator associated with $A_{a}^{i}$, and $F_{a b}^{i}$ is its associated curvature. Explicitly, $\mathcal{D}_{a} E_{i}^{a}=\partial_{a} E_{i}^{a}+\epsilon_{i j}{ }^{k} A_{a}^{j} E_{k}^{a}$ and $F_{a b}^{i}=2 \partial_{[a} A_{b]}^{i}+\epsilon_{j k}^{i} A_{a}^{j} A_{b}^{k}$, where $\partial_{a}$ is some fixed, flat derivative operator that ignores internal indices. $\epsilon_{i j k}$ is the Levi-Civita symbol for the internal space. The constraints $(1 a, b, c)$ are called the Gauss, scalar, and vector constraints, respectively. Since they are polynomial in $A_{a}^{i}$ and $E_{i}^{a}$, this theory extends GR to incorporate degenerate spatial metrics $q q^{a b} \equiv E_{i}^{a} E^{b i}$.

To evaluate the PB algebra of the constraints, I will first define constraint functionals $\mathcal{G}_{\Lambda}, \mathcal{S}_{N}$, and $\mathcal{V}_{\vec{N}}$ by smearing $\mathcal{G}_{i}, \mathcal{S}$, and $\mathcal{V}_{a}$ with appropriate test fields on $\Sigma$. By doing this, we obtain real-valued (rather than tensor-valued) functions on phase space. Explicitly,

$$
\begin{aligned}
\mathcal{G}_{\Lambda} & \equiv \int_{\Sigma} \Lambda^{i} \mathcal{D}_{a} E_{i}^{a}=0 \\
\mathcal{S}_{N} & \equiv \int_{\Sigma} N \epsilon^{i j}{ }_{k} E_{i}^{a} E_{j}^{b} F_{a b}^{k}=0 \\
\mathcal{V}_{\vec{N}} & \equiv \int_{\Sigma} N^{a} E_{j}^{b} F_{a b}^{j}=0
\end{aligned}
$$

where, for instance, $N$ is a scalar density of weight -1 . With this choice for $N$, the integrand of Eq. ( $2 b)$ is a scalar density of +1 , which can be integrated over $\Sigma$ without the need of any additional structure. Since we will concentrate only on the constraint algebra and not on dynamics, we can assume for asymptotically flat $\Sigma$ that the smearing fields fall-off sufficiently fast so that the above constraint functionals are differentiable and boundary terms can be ignored. For spatially compact $\Sigma$, these issues do not arise since $\partial \Sigma=0$.

As shown in [1], the PB algebra of the constraints is closed. That is, the PB of any two constraint functionals, Eqs. $(2 a, b, c)$, vanish modulo themselves. Explicitly, if one takes $\left\{E_{i}^{a}(x), A_{b}^{j}(y)\right\}=\delta_{i}^{j} \delta_{b}^{a} \delta^{3}(x, y)$ as the fundamental PB, one can show that

$$
\begin{aligned}
& \left\{\mathcal{G}_{\Lambda}, \mathcal{G}_{\Lambda^{\prime}}\right\}=-\mathcal{G}_{\left[\Lambda, \Lambda^{\prime}\right]} \approx 0 \\
& \left\{\mathcal{G}_{\Lambda}, \mathcal{V}_{\vec{M}}\right\}=0 \\
& \left\{\mathcal{G}_{\Lambda}, \mathcal{S}_{M}\right\}=0
\end{aligned}
$$




$$
\begin{aligned}
& \left\{\mathcal{V}_{\vec{N}}, \mathcal{V}_{\vec{M}}\right\}=-\mathcal{V}_{[\vec{N}, \vec{M}]}+\mathcal{G}_{N^{a} M^{b} F_{a b}} \approx 0 \\
& \left\{\mathcal{S}_{N}, \mathcal{V}_{\vec{M}}\right\}=\mathcal{S}_{\mathcal{L}_{\vec{M}} N}+\mathcal{G}_{2 N M^{a}\left[E^{b}, F_{a b}\right]} \approx 0 \\
& \left\{\mathcal{S}_{N}, \mathcal{S}_{M}\right\}=\mathcal{V}_{4 \vec{E}_{i} E^{b i}\left(N \partial_{b} M-M \partial_{b} N\right)} \approx 0
\end{aligned}
$$

where $\left[\Lambda, \Lambda^{\prime}\right] \equiv \epsilon_{j k}^{i} \Lambda^{j} \Lambda^{k},[\vec{N}, \vec{M}]^{a} \equiv N^{b} \partial_{b} M^{a}-M^{b} \partial_{b} N^{a}$, and $\left[E^{b}, F_{a b}\right]^{i} \equiv \epsilon^{i j} E_{j}^{b} F_{a b}^{k}$. Since the RHSs of all these equations are weakly zero, there are no secondary constraints.

\section{Alternative degenerate extension of GR}

The alternative degenerate extension of GR originally discussed in [5] is defined by the same phase space variables $\left(A_{a}^{i}, E_{i}^{a}\right)$ as in Ashtekar's theory, but they are subject to a different set of constraints. The Gauss and scalar constraints Eqs. $(1 a, b)$ are the same, but the vector constraint $\mathcal{V}_{a}=0$ is replaced by

$$
\mathcal{W}_{i} \equiv E_{i}^{a} \mathcal{V}_{a}=E_{i}^{a} E_{j}^{b} F_{a b}^{j}=0 .
$$

$\mathcal{W}_{i}=0$ will be called the weak vector constraint since it is implied by, but does not in general imply, the original vector constraint $\mathcal{V}_{a}=0$. Since the weak vector constraint is polynomial in $A_{a}^{i}$ and $E_{i}^{a}$, the alternative theory defined by the primary constraints Eqs. $(1 a, b)$ and (4) also extends GR to incorporate degenerate spatial metrics. The constraints of the two theories are equivalent if and only if the triad vectors $E_{i}^{a}$ are non-degenerate.f

Unlike the PB algebra of Ashtekar's original theory, the PB algebra of the alternative degenerate extension is not closed [5]. The PBs of the Gauss and scalar constraint functionals weakly vanish modulo the Gauss and weak vector constraints (see Eqs. $(3 a, c, f)$ ), but the PBs involving the weak vector constraint are not all weakly zero. Defining

$$
\mathcal{W}_{N} \equiv \int_{\Sigma} N^{i} E_{i}^{a} E_{j}^{b} F_{a b}^{j}=0
$$

\footnotetext{
${ }^{4}$ In $S L(2, C)$ spinor notation (see [1] or [0]), the phase variables are denoted by $A_{a}{ }^{A B}$ and $\sigma^{a}{ }_{A B}$, and the scalar and vector constraints $(1 b, c)$ have the form $\mathcal{S} \equiv \operatorname{Tr}\left(\sigma^{a} \sigma^{b} F_{a b}\right)=0$ and $\mathcal{V}_{a} \equiv \operatorname{Tr}\left(\sigma^{b} F_{a b}\right)=0$. These can be written as a combined constraint $\mathcal{C}_{A B} \equiv\left(\sigma^{a} \sigma^{b} F_{a b}\right)_{A B}=0$ with only internal indices, which in terms of $\mathcal{V}_{a}$ and $\mathcal{S}$ equals$$
\mathcal{C}_{A B}=\frac{1}{2} \epsilon_{A B} \mathcal{S}+\sigma^{a}{ }_{A B} \mathcal{V}_{a}
$$

Thus, $\mathcal{C}_{A B}=0$ if and only if $\mathcal{S}=0$ and $\mathcal{W}_{A B} \equiv \sigma^{a}{ }_{A B} \mathcal{V}_{a}=0$. The constraint $\mathcal{W}_{A B}=0$ corresponds to the constraint $\mathcal{W}_{i}=0$ in the triad notation of this paper. One had originally hoped that by writing the four diffeomorphism constraints in this combined form, the PB algebra of the constraints would simplify. Such a simplification occurs in $2+1$ gravity; the PB algebra there being $I S O(2,1)$. Unfortunately, such a simplification does not occur for the $3+1$ theory, even if one restricts attention to non-degenerate triad vectors [5].
} 
where $N^{i}$ is a smearing field of density weight -1 , one finds

$$
\begin{aligned}
\left\{\mathcal{W}_{N}, \mathcal{W}_{M}\right\} & \approx \mathcal{V}_{[\vec{N}(E), \vec{M}(E)]} \\
\left\{\mathcal{S}_{N}, \mathcal{W}_{M}\right\} & \approx \mathcal{V}_{N M^{i} \epsilon_{i}{ }^{j k}\left[\vec{E}_{j}, \vec{E}_{k}\right]} \\
\left\{\mathcal{G}_{\Lambda}, \mathcal{W}_{M}\right\} & \approx 0
\end{aligned}
$$

where $\vec{N}(E)$ and $\vec{M}(E)$ denote the field-dependent vector fields $N^{a}(E) \equiv N^{i} E_{i}^{a}$ and $M^{a}(E) \equiv$ $M^{i} E_{i}^{a}$, and $\left[E_{i}, E_{j}\right]^{a} \equiv E_{i}^{b} \partial_{b} E_{j}^{a}-E_{j}^{b} \partial_{b} E_{i}^{a}$ is the commutator of $E_{i}^{a}$ and $E_{j}^{a}$. Equations (6) hold modulo the Gauss, scalar, and weak vector constraints. (See the appendix of this paper or [5] for a detailed calculation of the above PBs.) The RHS of Eqs. (6) are weakly zero for all smearing fields if and only if

$$
\mathcal{W}_{i j} \equiv\left[E_{i}, E_{j}\right]^{a} \mathcal{V}_{a} \approx 0
$$

This is a secondary constraint.

Since the primary constraints are not closed under PB, we must repeat the above process. That is, we must also require that the $\mathrm{PBs}$ of $\mathcal{W}_{N}, \mathcal{S}_{N}$, and $\mathcal{G}_{\Lambda}$ with the secondary constraint Eq. (7) be weakly equal to zero if we eventually hope to obtain a consistent theory with a closed PB algebra. Since the constraint functional

$$
\int_{\Sigma} N^{i j} \mathcal{W}_{i j} \equiv \int_{\Sigma} N^{i j}\left[E_{i}, E_{j}\right]^{a} \mathcal{V}_{a}
$$

can be written as a sum of terms, each of the form

$$
\int_{\Sigma} M^{[i} L^{j]} \mathcal{W}_{i j}=\int_{\Sigma} M^{i} L^{j}\left[E_{i}, E_{j}\right]^{a} \mathcal{V}_{a} \approx \mathcal{V}_{[\vec{M}(E), \vec{L}(E)]},
$$

it suffices to calculate the PBs of $\mathcal{W}_{N}, \mathcal{S}_{N}$, and $\mathcal{G}_{\Lambda}$ with $\mathcal{V}_{[\vec{M}(E), \vec{L}(E)]}$. Evaluating these higher-order PBs leads to what I will call a higher-order secondary constraint. Explicitly, one finds

$$
\begin{aligned}
\left\{\mathcal{W}_{N}, \mathcal{V}_{[\vec{M}(E), \vec{L}(E)]}\right\} & \approx \mathcal{V}_{[\vec{N}(E),[\vec{M}(E), \vec{L}(E)]]} \\
\left\{\mathcal{S}_{N}, \mathcal{V}_{[\vec{M}(E), \vec{L}(E)]}\right\} & \approx \mathcal{V}_{N\left(M^{i} L^{l}-L^{i} M^{l}\right) \epsilon_{l} j k\left[\vec{E}_{i},\left[\vec{E}_{j}, \vec{E}_{k}\right]\right]} \\
\left\{\mathcal{G}_{\Lambda}, \mathcal{V}_{[\vec{M}(E), \vec{L}(E)]}\right\} & \approx 0
\end{aligned}
$$

where $\approx 0$ now means $=0$ modulo $\mathcal{G}_{i}=0, \mathcal{S}=0, \mathcal{W}_{i}=0$, and $\mathcal{W}_{i j}=0$. (Again, see the appendix for details involving the calculation of the above PBs.) The RHS of the above equations are weakly zero for all smearing fields if and only if

$$
\mathcal{W}_{i j k} \equiv\left[E_{i},\left[E_{j}, E_{k}\right]\right]^{a} \mathcal{V}_{a} \approx 0
$$

\footnotetext{
${ }^{5}$ The gauge dependence of this so-called commutator of vector densities will be discussed below, in the second-to-last paragraph of this section.
} 
This is a tertiary, or third-order, constraint. The explicit expression Eq. (11) for $\mathcal{W}_{i j k}$ is a new result that was not known in [5]. By repeating this procedure, one finds $\mathcal{W}_{i j k l} \approx 0, \cdots$, where $\mathcal{W}_{i j k l}, \cdots$, are the higher-order generalizations of Eqs. (7) and (11).

Two remarks are in order. The first involves what I have been calling the commutator of the triad vectors $E_{i}^{a}$ and $E_{j}^{a}$. As noted in the sentence immediately following Eqs. (6), $\left[E_{i}, E_{j}\right]^{a}$ is defined by

$$
\left[E_{i}, E_{j}\right]^{a} \equiv E_{i}^{b} \partial_{b} E_{j}^{a}-E_{j}^{b} \partial_{b} E_{i}^{a}
$$

where $\partial_{a}$ is the fixed, flat derivative operator that we originally used to define $\mathcal{D}_{a}$ in terms of $A_{a}^{i}$. Since $E_{i}^{a}$ are vector densities of weight +1 , Eq. (12) depends on the choice of $\partial_{a}$. In this sense, the commutator of the triad vectors is gauge dependent. Nonetheless, the constraints $\mathcal{W}_{i j} \approx 0, \mathcal{W}_{i j k} \approx 0, \cdots$, are independent of the choice of $\partial_{a}$ modulo previous constraints. Moreover, $\left[E_{i}, E_{j}\right]^{a}=C^{k}{ }_{i j} E_{k}^{a}$ has the same geometrical interpretation as surface-forming vector fields, again independent of the the choice of $\partial_{a}$. This is because the commutator $\left[E_{i}, E_{j}\right]^{a}$ picks up terms proportional to $E_{i}^{a}$ and $E_{j}^{a}$ under a change of derivative operator.

The second remark is that the totality of constraints for the alternative degenerate extension of GR is first class. This was proved in [5] using the Jacobi identity

$$
\{f,\{g, h\}\}+\{g,\{h, f\}\}+\{h,\{f, g\}\}=0
$$

for PBs. In fact, the proof given there showed that the first class nature of the constraints

does not depend on the explicit form of the higher-order secondary constraints. As an illustration of this general result, consider the PB of two secondary constraints $\mathcal{V}_{[\vec{N}(E), \vec{M}(E)]}$ and $\mathcal{V}_{[\vec{L}(E), \vec{K}(E)]}$. Using Eqs. (13), (6a), (10a), and their higher-order generalizations, we find

$$
\begin{aligned}
\left\{\mathcal{V}_{[\vec{N}(E), \vec{M}(E)]}, \mathcal{V}_{[\vec{L}(E), \vec{K}(E)]}\right\} & \approx\left\{\left\{\mathcal{W}_{N}, \mathcal{W}_{M}\right\},\left\{\mathcal{W}_{L}, \mathcal{W}_{K}\right\}\right\} \\
& =\left\{\mathcal{W}_{K},\left\{\mathcal{W}_{L},\left\{\mathcal{W}_{N}, \mathcal{W}_{M}\right\}\right\}-\left\{\mathcal{W}_{L},\left\{\mathcal{W}_{K},\left\{\mathcal{W}_{N}, \mathcal{W}_{M}\right\}\right\}\right.\right. \\
& \approx \mathcal{V}_{[\vec{K}(E),[\vec{L}(E),[\vec{N}(E), \vec{M}(E)]]]}-L \leftrightarrow K
\end{aligned}
$$

modulo first, second, and third-order constraints. Thus, the PB of two secondary constraints is weakly equal to a sum of fourth-order constraints.

\section{Generic classification: Redundancy of the higher-order constraints}

We are now ready to address the question regarding the redundancy of the higher-order secondary constraints for the alternative degenerate extension of GR. In the process of answering it, we will obtain a generic classification of the difference between the alternative theory and Ashtekar's original degenerate extension of GR. As suggested by Eqs. (7), (11), 
and their higher-order generalizations, the relationship between the two theories depends on the degeneracy and surface-forming properties of the triad vectors.

Let $x \in \Sigma$ be any point of space, and assume that the degeneracy and surface-forming properties of the triad vectors is the same in an open set about $x$. Then we have the following generic classification:

1. If the triad vectors are non-degenerate, then $\mathcal{W}_{i}=0$ is equivalent to $\mathcal{V}_{a}=0$, and $\mathcal{W}_{i j}=0, \cdots$, are automatically satisfied. There is nothing surprising here. We already knew that both theories agree in the non-degenerate sector.

2. If the triad vectors are degenerate and surface-forming, then although $\mathcal{W}_{i}=0$ does not imply $\mathcal{V}_{a}=0$, the secondary constraints $\mathcal{W}_{i j}=0, \cdots$, are automatically satisfied as a consequence of $\mathcal{W}_{i}=0$. This is because $\left[E_{i}, E_{j}\right]^{a}, \cdots$, are proportional to $E_{k}^{a}$, and $E_{k}^{a} \mathcal{V}_{a}=\mathcal{W}_{k}=0$. Thus, the alternative degenerate extension of GR is different from Ashtekar's original degenerate extension.

3. If the triad vectors are degenerate but are not surface-forming, then $\mathcal{W}_{i}=0$ and the first secondary constraint $\mathcal{W}_{i j}=0$ already imply $\mathcal{V}_{a}=0$. (The higher-order secondary constraints $\mathcal{W}_{i j k}=0, \cdots$, are automatically satisfied as well.) This is because $E_{i}^{a}$ and $\left[E_{i}, E_{j}\right]^{a}$ are linearly independent, so $\mathcal{W}_{i} \equiv E_{i}^{a} \mathcal{V}_{a}=0$ and $\mathcal{W}_{i j} \equiv\left[E_{i}, E_{j}\right]^{a} \mathcal{V}_{a}=0$ imply $\mathcal{V}_{a}=0$. Thus, for this case, the alternative degenerate extension of GR is equivalent to Ashtekar's theory.

As explicit example of case 2 above, one can consider

$$
E_{i}^{a} \equiv\left((\partial / \partial x)^{a},(\partial / \partial y)^{a}, 0\right) \quad \text { and } \quad A_{a}^{i} \equiv\left(x(d z)_{a}, y(d z)_{a}, 0\right)
$$

where $(x, y, z)$ are coordinates about some point in space. For such initial data, $\mathcal{G}_{i}=0$, $\mathcal{S}=0, \mathcal{W}_{i}=0, \mathcal{W}_{i j}=0, \cdots$, but $\mathcal{V}_{a}=-2(d z)_{a} \neq 0$. Thus, these phase space variables are a solution to the alternative theory, but fail to be a solution of Ashtekar's theory.?

\section{Conclusion}

By finding an explicit expression for the higher-order secondary constraints of the alternative degenerate extension of GR, we have been able to resolve the puzzle of the "infinite"

\footnotetext{
${ }^{6}$ Under the infinitesimal evolution generated by the scalar constraint functional $\mathcal{S}_{N}$, one finds $\dot{A}_{a}^{i}=0$ (for $i=1,2,3), \dot{E}_{1}^{a}=0, \dot{E}_{2}^{a}=0$, and $\dot{E}_{3}^{a}=2(\partial N / \partial y)(\partial / \partial x)^{a}-2(\partial N / \partial x)(\partial / \partial y)^{a}$, where $\dot{f} \equiv\left\{\mathcal{S}_{N}, f\right\}$. Thus, for $N$ independent of $x$ and $y$ (in particular, for $N=1$ ), this degenerate solution, Eq. (15), of the initial value constraints of the alternative theory is constant in time.
} 
tower of secondary constraints originally raised in [5]. From Eqs. (7), (11), etc. one sees that the secondary constraints and their higher-order generalizations are nothing more than the vector constraint of Ashtekar's original degenerate extension contracted with nested commutators of the triad vectors. Whenever the triad vectors and any of the nested commutators span the tangent space at a point in space, then $\mathcal{W}_{i}=0, \mathcal{W}_{i j}=0, \cdots$, imply $\mathcal{V}_{a}=0$ and the two theories are equivalent. More generally, the classification given in section 4 completely characterizes the difference between the two degenerate extensions of GR when the degeneracy and surface-forming properties of triad vectors is the same everywhere in an open set about a point in space.

But what about those cases where the degeneracy and surface-forming properties of the triad vectors change from point to point? What can one say about the relationship between the two degenerate extensions in these cases? Unfortunately, I do not know the general answer to these questions at present. Only when the triad vectors are continuous and degenerate on a set of measure zero, can I make a definite statement. For such a triad, the two theories are equivalent. This is because $\mathcal{W}_{i} \equiv E_{i}^{a} \mathcal{V}_{a}=0$ implies $\mathcal{V}_{a}=0$ wherever $E_{i}^{a}$ is non-degenerate, while continuity of $\mathcal{V}_{a}$ implies $\mathcal{V}_{a}=0$ elsewhere. The classification for the opposite case (where the triad vectors have support only on a set of measure zero) is yet to be understood.

\section{ACKNOWLEDGEMENTS}

I would like to thank Ted Jacobson for the numerous discussions I had with him throughout the course of this work. I would also like to thank Riccardo Capovilla, Jonathan Simon, and Ranjeet Tate for their helpful remarks, especially related to the calculation of the PBs for the alternative degenerate extension. This work was supported by NSF grant PHY91-12240.

\section{Appendix: Calculation of the PBs for the alternative degenerate extension}

In this appendix, I will describe in detail a method of calculating the PBs of $\mathcal{W}_{N}, \mathcal{S}_{N}$, and $\mathcal{G}_{\Lambda}$ with the weak vector constraint $\mathcal{W}_{M}$ of the alternative degenerate extension of GR. This method of calculation takes advantage of the PBs (Eqs. (3)) for Ashtekar's original degenerate extension. This method of calculation can also be generalized to give the PBs of $\mathcal{W}_{N}, \mathcal{S}_{N}$, and $\mathcal{G}_{\Lambda}$ with the secondary constraint functional $\mathcal{V}_{[\vec{M}(E), \vec{L}(E)]}$ and with all higherorder secondary constraints. (The necessary steps are sketched in the final paragraph of the appendix.) Since we will be interested only in obtaining a closed PB algebra for the alternative degenerate extension, we will evaluate the PBs modulo previous constraints. We 
will not find explicit expressions for the structure functions of this theory.

The key to this method of calculation is the equality $\mathcal{W}_{M}=\mathcal{V}_{\vec{M}(E)}$. Using this equality, we can write

$$
\begin{aligned}
& \frac{\delta \mathcal{W}_{M}}{\delta E_{i}^{a}}=\left.\frac{\delta \mathcal{V}_{\vec{M}}}{\delta E_{i}^{a}}\right|_{\vec{M}(E)}+\mathcal{V}_{c} \frac{\partial M^{c}(E)}{\partial E_{i}^{a}} \\
& \frac{\delta \mathcal{W}_{M}}{\delta A_{a}^{i}}=\left.\frac{\delta \mathcal{V}_{\vec{M}}}{\delta A_{a}^{i}}\right|_{\vec{M}(E)}
\end{aligned}
$$

where $\left.\left(\delta \mathcal{V}_{\vec{M}} / \delta E_{i}^{a}\right)\right|_{\vec{M}(E)}$ denotes the variation of $\mathcal{V}_{\vec{M}(E)}$ with respect to $E_{i}^{a}$ holding $A_{a}^{i}$ fixed and treating $M^{a}(E) \equiv M^{i} E_{i}^{a}$ as if it were independent of $E_{i}^{a}$. A similar definition applies to $\left.\left(\delta \mathcal{V}_{\vec{M}} / \delta A_{a}^{i}\right)\right|_{\vec{M}(E)}$. Thus,

$$
\begin{aligned}
\left\{\mathcal{W}_{N}, \mathcal{W}_{M}\right\} & \equiv\left\{\mathcal{V}_{\vec{N}(E)}, \mathcal{V}_{\vec{M}(E)}\right\} \\
& =\int_{\Sigma} \frac{\delta \mathcal{W}_{N}}{\delta E_{i}^{a}} \frac{\delta \mathcal{W}_{M}}{\delta A_{a}^{i}}-\frac{\delta \mathcal{W}_{M}}{\delta E_{i}^{a}} \frac{\delta \mathcal{W}_{N}}{\delta A_{a}^{i}} \\
& =\left.\int_{\Sigma}\left(\left.\frac{\delta \mathcal{V}_{\vec{N}}}{\delta E_{i}^{a}}\right|_{\vec{N}(E)}+\mathcal{V}_{c} \frac{\partial N^{c}(E)}{\partial E_{i}^{a}}\right) \frac{\delta \mathcal{V}_{\vec{M}}}{\delta A_{a}^{i}}\right|_{\vec{M}(E)}-N \leftrightarrow M \\
& =\left\{\left.\mathcal{V}_{\vec{N}}\right|_{\vec{N}(E)},\left.\mathcal{V}_{\vec{M}}\right|_{\vec{M}(E)}\right\}+\int_{\Sigma}\left(\left.\mathcal{V}_{c} \frac{\partial N^{c}(E)}{\partial E_{i}^{a}} \frac{\delta \mathcal{V}_{\vec{M}}}{\delta A_{a}^{i}}\right|_{\vec{M}(E)}-N \leftrightarrow M\right)
\end{aligned}
$$

where the first term on the last line denotes the PB of $\mathcal{V}_{\vec{N}(E)}$ and $\mathcal{V}_{\vec{M}(E)}$ treating $\vec{N}(E)$ and $\vec{M}(E)$ as if they were independent of $E_{i}^{a}$. Similarly,

$$
\begin{aligned}
& \left\{\mathcal{S}_{N}, \mathcal{W}_{M}\right\}=\left\{\mathcal{S}_{N},\left.\mathcal{V}_{\vec{M}}\right|_{\vec{M}(E)}\right\}-\int_{\Sigma} \mathcal{V}_{c} \frac{\partial M^{c}(E)}{\partial E_{i}^{a}} \frac{\delta \mathcal{S}_{N}}{\delta A_{a}^{i}} \\
& \left\{\mathcal{G}_{\Lambda}, \mathcal{W}_{M}\right\}=\left\{\mathcal{G}_{\Lambda},\left.\mathcal{V}_{\vec{M}}\right|_{\vec{M}(E)}\right\}-\int_{\Sigma} \mathcal{V}_{c} \frac{\partial M^{c}(E)}{\partial E_{i}^{a}} \frac{\delta \mathcal{G}_{\Lambda}}{\delta A_{a}^{i}}
\end{aligned}
$$

To evaluate the above PBs (Eqs. $(A .2 a, b, c)$ ), it is useful to note that the first two terms on the last line of Eq. $(A .2 a)$ weakly cancel. This cancellation holds modulo the Gauss and weak vector constraints. To see this, recall that (see Eq. $(3 d)$ )

$$
\begin{aligned}
\left\{\left.\mathcal{V}_{\vec{N}}\right|_{\vec{N}(E)},\left.\mathcal{V}_{\vec{M}}\right|_{\vec{M}(E)}\right\} & =-\mathcal{V}_{[\vec{N}(E), \vec{M}(E)]}+\mathcal{G}_{N^{a}(E) M^{b}(E) F_{a b}} \\
& \approx-\mathcal{V}_{[\vec{N}(E), \vec{M}(E)]}
\end{aligned}
$$

In addition,

$$
\left.\frac{\delta \mathcal{V}_{\vec{M}}}{\delta A_{a}^{i}}\right|_{\vec{M}(E)}=2 \mathcal{D}_{b}\left(M^{[a}(E) E_{i}^{b]}\right)
$$


If we expand the covariant derivative in Eq. (A.4) using the Leibnitz rule and contract the resulting expression with $\partial N^{c}(E) / \partial E_{i}^{a}=\delta_{a}^{c} N^{i}$, we find

$$
\left.\frac{\partial N^{c}(E)}{\partial E_{i}^{a}} \frac{\delta \mathcal{V}_{\vec{M}}}{\delta A_{a}^{i}}\right|_{\vec{M}(E)}=[N(E), M(E)]^{c}+\left(\text { terms proportional to } \mathcal{G}_{i} \text { and } E_{i}^{c}\right) .
$$

Thus,

$$
\left.\int_{\Sigma} \mathcal{V}_{c} \frac{\partial N^{c}(E)}{\partial E_{i}^{a}} \frac{\delta \mathcal{V}_{\vec{M}}}{\delta A_{a}^{i}}\right|_{\vec{M}(E)} \approx \mathcal{V}_{[\vec{N}(E), \vec{M}(E)]}
$$

modulo the Gauss and weak vector constraints. Combining Eqs. (A.3) and (A.6), we obtain

$$
\left\{\mathcal{W}_{N}, \mathcal{W}_{M}\right\} \approx-\left.\int_{\Sigma} \mathcal{V}_{c} \frac{\partial M^{c}(E)}{\partial E_{i}^{a}} \frac{\delta \mathcal{V}_{\vec{N}}}{\delta A_{a}^{i}}\right|_{\vec{N}(E)}
$$

Similarly,

$$
\begin{aligned}
& \left\{\mathcal{S}_{N}, \mathcal{W}_{M}\right\} \approx-\int_{\Sigma} \mathcal{V}_{c} \frac{\partial M^{c}(E)}{\partial E_{i}^{a}} \frac{\delta \mathcal{S}_{N}}{\delta A_{a}^{i}} \\
& \left\{\mathcal{G}_{\Lambda}, \mathcal{W}_{M}\right\}=-\int_{\Sigma} \mathcal{V}_{c} \frac{\partial M^{c}(E)}{\partial E_{i}^{a}} \frac{\delta \mathcal{G}_{\Lambda}}{\delta A_{a}^{i}}
\end{aligned}
$$

These hold modulo the Gauss and scalar constraints. (See Eqs. (3e) and (3b).)

To complete the calculation of the $\mathrm{PBs}$, we need to evaluate various functional derivatives. These include

$$
\begin{aligned}
& \left.\frac{\delta \mathcal{V}_{\vec{N}}}{\delta A_{a}^{i}}\right|_{\vec{N}(E)}=2 \mathcal{D}_{b}\left(N^{[a}(E) E_{i}^{b]}\right) \\
& \frac{\delta \mathcal{S}_{N}}{\delta A_{a}^{i}}=2 \epsilon_{i}{ }^{j k} \mathcal{D}_{b}\left(N E_{j}^{[a} E_{k}^{b]}\right) \\
& \frac{\delta \mathcal{G}_{\Lambda}}{\delta A_{a}^{i}}=-\Lambda^{j} \epsilon_{i j}{ }^{k} E_{k}^{a} .
\end{aligned}
$$

Since $M^{a} \equiv M^{i} E_{i}^{a}$, we also have

$$
\frac{\partial M^{c}(E)}{\partial E_{i}^{a}} T_{i}^{a}=M^{i} T_{i}^{c} \equiv M^{c}(T)
$$

for any $T_{i}^{a}$ of density-weight +1 . If we take $T_{i}^{a}$ to be the above functional derivatives, Eqs. (A.8), and expand the covariant derivative in Eqs. $(A .8 a)$ and $(A .8 b)$, we find

$$
\begin{aligned}
& \left.\frac{\partial M^{c}(E)}{\partial E_{i}^{a}} \frac{\delta \mathcal{V}_{\vec{N}}}{\delta A_{a}^{i}}\right|_{\vec{N}(E)}=-[N(E), M(E)]^{c}+\left(\text { terms proportional to } \mathcal{G}_{i} \text { and } E_{i}^{c}\right) \\
& \frac{\partial M^{c}(E)}{\partial E_{i}^{a}} \frac{\delta \mathcal{S}_{N}}{\delta A_{a}^{i}}=-N M^{i} \epsilon_{i}{ }^{j k}\left[E_{j}, E_{k}\right]^{c}+\left(\text { terms proportional to } \mathcal{G}_{i} \text { and } E_{i}^{c}\right) \\
& \frac{\partial M^{c}(E)}{\partial E_{i}^{a}} \frac{\delta \mathcal{G}_{\Lambda}}{\delta A_{a}^{i}}=\left(\text { terms proportional to } E_{i}^{c}\right)
\end{aligned}
$$


Thus,

$$
\begin{aligned}
\left\{\mathcal{W}_{N}, \mathcal{W}_{M}\right\} & \approx \mathcal{V}_{[\vec{N}(E), \vec{M}(E)]} \\
\left\{\mathcal{S}_{N}, \mathcal{W}_{M}\right\} & \approx \mathcal{V}_{N M^{i} \epsilon_{i}{ }^{j k}\left[\vec{E}_{j}, \vec{E}_{k}\right]} \\
\left\{\mathcal{G}_{\Lambda}, \mathcal{W}_{M}\right\} & \approx 0
\end{aligned}
$$

modulo the Gauss, scalar, and weak vector constraints. These are Eqs. (6) in the main text.]

To evaluate the PBs of $\mathcal{W}_{N}, \mathcal{S}_{N}$, and $\mathcal{G}_{\Lambda}$ with the secondary constraint functional $\mathcal{V}_{[\vec{M}(E), \vec{L}(E)]}$, we can basically proceed as above. We simply replace $M^{a}(E)$ with the commutator $[M(E), L(E)]^{a}$ and calculate the PBs modulo $\mathcal{G}_{i}=0, \mathcal{S}=0, \mathcal{W}_{i}=0$, and $\mathcal{W}_{i j} \equiv\left[E_{i}, E_{j}\right]^{a} \mathcal{V}_{a}=0$. We are allowed to make this simple replacement since Eqs. $(A .7)$, when written in terms of $\mathcal{V}_{\vec{M}(E)}$,

$$
\begin{aligned}
\left\{\mathcal{W}_{N}, \mathcal{V}_{\vec{M}(E)}\right\} & \approx-\left.\int_{\Sigma} \mathcal{V}_{c} \frac{\partial M^{c}(E)}{\partial E_{i}^{a}} \frac{\delta \mathcal{V}_{\vec{N}}}{\delta A_{a}^{i}}\right|_{\vec{N}(E)} \\
\left\{\mathcal{S}_{N}, \mathcal{V}_{\vec{M}(E)}\right\} & \approx-\int_{\Sigma} \mathcal{V}_{c} \frac{\partial M^{c}(E)}{\partial E_{i}^{a}} \frac{\delta \mathcal{S}_{N}}{\delta A_{a}^{i}} \\
\left\{\mathcal{G}_{\Lambda}, \mathcal{V}_{\vec{M}(E)}\right\} & =-\int_{\Sigma} \mathcal{V}_{c} \frac{\partial M^{c}(E)}{\partial E_{i}^{a}} \frac{\delta \mathcal{G}_{\Lambda}}{\delta A_{a}^{i}}
\end{aligned}
$$

hold for any field-dependent smearing field $\vec{M}(E)$. In particular, Eqs. $(A .12)$ are valid when $\vec{M}(E)$ is replaced by the commutator $[\vec{M}(E), \vec{L}(E)]$. In addition, Eq. $(A .9)$ should be replaced by

$$
\begin{aligned}
\frac{\partial[M(E), L(E)]^{c}}{\partial E_{i}^{a}} T_{i}^{a} & =\left[\frac{\partial M(E)}{\partial E_{i}^{a}} T_{i}^{a}, L(E)\right]^{c}+\left[M(E), \frac{\partial L(E)}{\partial E_{i}^{a}} T_{i}^{a}\right]^{c} \\
& \equiv[M(T), L(E)]^{c}+[M(E), L(T)]^{c}
\end{aligned}
$$

which together with Eqs. (A.10) imply

$$
\begin{aligned}
& \left.\frac{\partial[M(E), L(E)]^{c}}{\partial E_{i}^{a}} \frac{\delta \mathcal{V}_{\vec{N}}}{\delta A_{a}^{i}}\right|_{\vec{N}(E)}=-[N(E),[M(E), L(E)]]^{c} \\
& +\left(\text { terms proportional to } \mathcal{G}_{i}, E_{i}^{c} \text {, and }\left[E_{i}, E_{j}\right]^{c}\right) \\
& \frac{\partial[M(E), L(E)]^{c}}{\partial E_{i}^{a}} \frac{\delta \mathcal{S}_{N}}{\delta A_{a}^{i}}=-N\left(M^{i} L^{l}-L^{i} M^{l}\right) \epsilon_{l}{ }^{j k}\left[E_{i},\left[E_{j}, E_{k}\right]\right]^{c}
\end{aligned}
$$

\footnotetext{
${ }^{7}$ Since the Gauss constraint generates internal gauge transformations, one could have immediately written down $\left\{\mathcal{G}_{\Lambda}, \mathcal{W}_{M}\right\}=-\mathcal{W}_{[\Lambda, M]}$, where $[\Lambda, M]^{i} \equiv \epsilon^{i}{ }_{j k} \Lambda^{j} M^{k}$. Thus, $\left\{\mathcal{G}_{\Lambda}, \mathcal{W}_{M}\right\} \approx 0$ modulo the weak vector constraint. In fact, the $\mathrm{PB}$ of the Gauss constraint with any constraint $\mathcal{C}$ is either identically zero (if $\mathcal{C}$ is gauge invariant) or weakly zero modulo $\mathcal{C}=0$ (if $\mathcal{C}$ has internal indices).
} 


$$
\begin{aligned}
&+\left(\text { terms proportional to } \mathcal{G}_{i}, E_{i}^{c} \text {, and }\left[E_{i}, E_{j}\right]^{c}\right) \\
& \frac{\partial[M(E), L(E)]^{c}}{\partial E_{i}^{a}} \frac{\delta \mathcal{G}_{\Lambda}}{\delta A_{a}^{i}}=\left(\text { terms proportional to } E_{i}^{c} \text { and }\left[E_{i}, E_{j}\right]^{c}\right) .
\end{aligned}
$$

Contracting the above expressions with $\mathcal{V}_{c}$ and integrating over $\Sigma$, we find

$$
\begin{aligned}
\left\{\mathcal{W}_{N}, \mathcal{V}_{[\vec{M}(E), \vec{L}(E)]}\right\} & \approx \mathcal{V}_{[\vec{N}(E),[\vec{M}(E), \vec{L}(E)]]} \\
\left\{\mathcal{S}_{N}, \mathcal{V}_{[\vec{M}(E), \vec{L}(E)]}\right\} & \approx \mathcal{V}_{N\left(M^{i} L^{l}-L^{i} M^{l}\right) \epsilon^{j k}\left[\vec{E}_{i},\left[\vec{E}_{j}, \vec{E}_{k}\right]\right]} \\
\left\{\mathcal{G}_{\Lambda}, \mathcal{V}_{[\vec{M}(E), \vec{L}(E)]}\right\} & \approx 0
\end{aligned}
$$

modulo $\mathcal{G}_{i}=0, \mathcal{S}=0, \mathcal{W}_{i}=0$, and $\mathcal{W}_{i j}=0$. These are Eqs. (10) in the main text. The PBs of $\mathcal{W}_{N}, \mathcal{S}_{N}$, and $\mathcal{G}_{\Lambda}$ with the higher-order secondary constraints $\mathcal{V}_{[\vec{M}(E),[\vec{L}(E), \vec{K}(E)]]}, \cdots$, are obtained in a similar manner. 


\section{References}

[1] A. Ashtekar, Phys. Rev. Lett. 57, 2244 (1986); Phys. Rev. D36, 1587 (1987).

[2] E. Witten, Nucl. Phys. B311, 46 (1988).

[3] G.T. Horowitz, Class. and Quantum Grav. 8, 587 (1991).

[4] A. Ashtekar, Lectures on Non-Perturbative Canonical Gravity, (Singapore: World Scientific), (1991).

[5] T. Jacobson and J.D. Romano, Class. and Quantum Grav. 9, L119 (1992). 